\title{
A new marker, SOX11, aids the diagnosis of mantle cell lymphoma in the prostate: A case report
}

\author{
BINGHAI CHEN ${ }^{1}$, GUANGMING YIN ${ }^{1}$, LUJING DUAN ${ }^{2}$, WANMENG LI $^{3}$ and XIANZHEN JIANG ${ }^{1}$ \\ ${ }^{1}$ Department of Urology, Third Xiang-Ya Hospital of Central South University, Yuelu, Changsha 410000; \\ ${ }^{2}$ Department of Endocrinology, Affiliated Hospital of Jiangsu University, Jingkou, Zhenjiang 210000; \\ ${ }^{3}$ Department of Radiology, Third Xiang-Ya Hospital of Central South University, Yuelu, Changsha 410000, P.R. China
}

Received January 26, 2012; Accepted May 18, 2012

DOI: $10.3892 / \mathrm{ol} .2012 .732$

\begin{abstract}
Mantle cell lymphoma (MCL) of the prostate, either primary or secondary, is a rare entity. This case report examines an 83-year-old male who complained of not only nocturia (2-3 times), but also frequency and urgency of urination. The maximal urinary flow rate was $4.1 \mathrm{ml} / \mathrm{sec}$. A transrectal ultrasound-guided prostate biopsy was advised after a hard enlargement of the prostate was detected; however, it was refused. Therefore, a plasma kinetic transurethral resection of the prostate was performed. Postoperative pathological examinations demonstrated MCL of the prostate. Positive immunohistochemistry for CD5 and cyclin D1 was observed. The diagnosis was confirmed by the introduction of a new diagnostic marker, SOX11. The maximal flow rate achieved was $15 \mathrm{ml} / \mathrm{sec}$ following surgery. To the best of our knowledge, this is the first study of MCL being diagnosed using SOX11 as a marker in the prostate. This case should alert clinicians and pathologists to pay close attention to the diagnosis of malignant lymphoma of the prostate. This study provides further insights into the diagnosis and therapy of MCL.
\end{abstract}

\section{Introduction}

Mantle cell lymphoma (MCL) is a subtype of B-cell non-Hodgkin's lymphoma. Patients diagnosed with MCL are usually elderly individuals and often present with stage III to IV. The involvement of extra-nodal tissues and organs, including Waldeyer's ring, bone marrow, peripheral blood, liver and the digestive tract, are common. MCL of the prostate, either primary or secondary, is a rare entity (1). According to three existing studies (2-4), the diagnosis of prostatic MCL is difficult and based on more extensive pathological analysis. Morphological and immunohistochemical investigations are

Correspondence to: Professor Xianzhen Jiang, Department of Urology, Third Xiang-Ya Hospital of Central South University, Tongzhipo Road, Yuelu, Changsha 410000, P.R. China

E-mail: jiangxzxy@yahoo.com.cn

Key words: mantle cell lymphoma, prostate, SOX11, cyclin D1 essential to diagnose MCL. The overexpression of CD5 and cyclin D1 are considered as markers of MCL, however, a definite diagnosis cannot be made in cases with negative or low expression of cyclin D1. This study presents a case which is unusual in its diagnostic method of MCL in the prostate and variety of diagnosis.

\section{Case report}

An 83-year-old male, who had a medical history of 3-year lower urinary tract symptoms (LUTS), complained of not only nocturia (2-3 times), but also frequency and urgency of urination. A urethral catheter was applied 20 days prior to hospitalization due to urinary retention and dysuria. The medical history of the patient included type II diabetes, hypertension commencing five years prior to presenting chronic obstructive pulmonary disease (COPD). The results of the clinical examinations were normal. The patient had no multiple non-tender subcutaneous nodules, and digital rectal examination revealed a smooth, firm, hard enlargement of the prostate. The patient reported no exposure to hazardous chemicals and had no family history of prostate cancer. The TPSA of this patient was $3.2 \mathrm{ng} / \mathrm{ml}$ and the maximal urinary flow rate was $4.1 \mathrm{ml} / \mathrm{sec}$. Ultrasound revealed a moderately enlarged nodular prostate and diffuse retroperitoneal lymph nodes. A pelvic MRI scan confirmed the prostate dimensions to be $43 \times 36 \times 35 \mathrm{~mm}$ and the lesion of the femural neck was of iso- or hypo-intensity on T1WI (arrow, Fig. 1A) and mixed signal intensity on T2WI without enhancement. The lesion of the femural neck was interpreted to be possible bone metastasis. Cystoscopy revealed a moderately large prostate and bladder tumor size of 4x3 mm. Transrectal ultrasound-guided prostate biopsy was refused and a plasma kinetic transurethral resection of the prostate and bladder tumor were therefore simultaneously performed. Postoperative pathological examinations demonstrated lymphoid follicles containing a wide variety of small-sized lymphocytes in the prostate (Fig. 1B). The lymphocytes were immunoreactive with CD20, CD5, SOX11 (Fig. 1C) and cyclin D1 (Fig. 1D), but negative results were obtained for CD3 and CD45RO. There were large glandular structures containing eosinophilic secretions. The extrusion and invasion of lymphocytes was not observed in the glandular cavity. It demonstrated transitional epithelial cells 

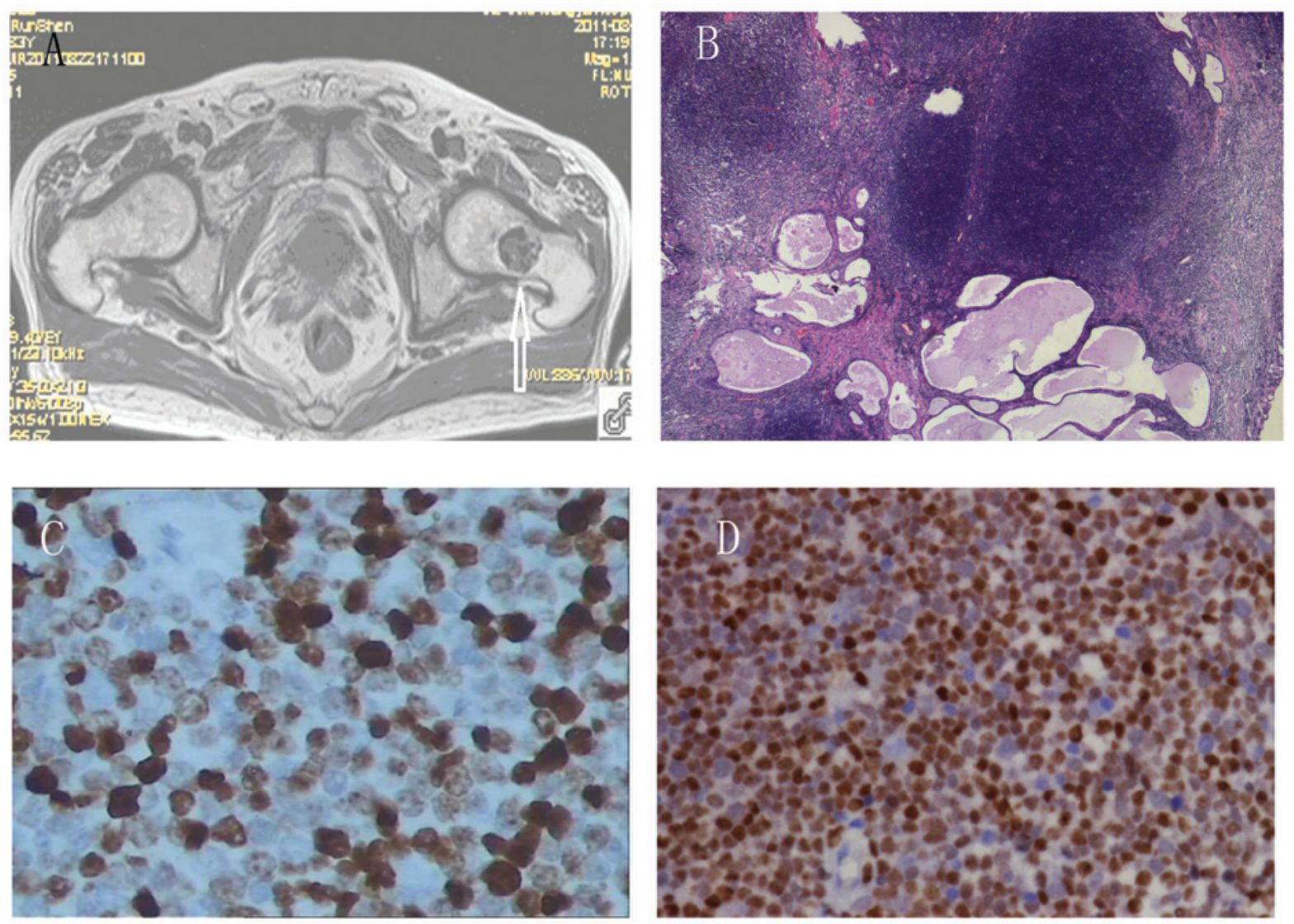

Figure 1. (A) Lesion of the left femural neck with iso- or hypo-intensity on T1WI. (B) Cytological appearance revealed lymphoid follicles containing a wide variety of small-sized lymphocytes in the prostate (H\&E staining; magnification, x200). (C) Representative immunohistochemical staining patterns for SOX11. Nuclei of lymphocytes were SOX11-positive (magnification, x400). (D) Immunohistochemical staining with cyclin D1 demonstrated lymphocytes with strong positivity (magnification, $\mathrm{x} 200$ ). H\&E, hematoxylin and eosin.

with atypical mitosis in the bladder tumor, however, the pathological results were unclear due to the small size of the tumor specimen. In a computed tomography scan of the chest, no positive result was determined and the diagnosis was accepted as a stage IV-A MCL without loss of weight, fever or night sweats. The patient was diagnosed with MCL of the prostate, bladder tumor, type II diabetes, hypertension and COPD.

\section{Discussion}

MCL is characterized by a proliferation of lymphocytes, with a high recurrence rate leading to mortality. An average survival time of three years was revealed (5). The median incidence of MCL is approximately 5 cases in every 100,000 individuals per year and the proportion of male to female cases is approximately 2.4:1 (6). It is well-known that the MCL histological types include diffuse, nodular and mantle zone. The typical cytological morphological feature of MCL is a diffuse infiltration of lymphocytes with an irregular nucleus and inconspicuous nucleolus. In this case, a large number of atypical lymphocytes, small-noncleaved and small-cleaved, are identified in lymphoid follicles. Tumor cell pleomorphism is not evident compared with that of normal lymphocytes. Lymphoid follicles are seldom observed in the prostate, therefore the suspected lymphoma was investigated by means of immunohistochemistry. In addition, a $3 \mu \mathrm{m}$ paraffin block is essential for MCL diagnosis, the efficiency of which is usually influenced by the distribution of atypical lymphocytes under the microscope. Due to the high degree of suspicion in this case, further pathological analysis was performed and positive immunohistochemistry for CD5 and cyclin D1 were observed. The overexpression of cyclin D1 was also observed in other cases with either chronic B lymphocytic leukemia or plasmocytoma and it was found that no less than $15 \%$ of cases with MCL were cyclin D1-negative (7). A new diagnostic marker, SOX11, was therefore introduced, which is positive in over $90 \%$ of MCL, but not in B-CLL or follicular lymphoma cases $(8,9)$. In patients with MCL, either cyclin D1-positive or cyclin D1-negative, the overexpression of SOX11 was observed, therefore SOX11 was suggested to be a diagnostic marker of MCL (8). We then analyzed the expression of SOX11. A positive expression of SOX11 confirmed the diagnosis of MCL in the present case. The differential diagnosis of LUTS in patients generally includes benign prostatic hyperplasia, overactive bladder and urinary tract infection, but not lymphomatous involvement, which is usually overlooked. In this case, urinary manifestations revealed progressive severe dysuria and urinary retention. The patient was therefore treated with plasma kinetic transurethral resection of the prostate, which is not usually performed for lymphoma. The maximal flow rate achieved was $15 \mathrm{ml} / \mathrm{sec}$ following surgery, however, the treatment of MCL is usually unsatisfactory. CHOP are used as conventional chemotherapies for MCL. Stem cell 
transplantation (SCT), either autologous or allogeneic, may ameliorate the prognosis, however, it is difficult to overcome the application limitations of SCT due to MCL relapse and high toxicity $(10,11)$.

This case should alert the clinician and pathologist to pay close attention to the diagnosis of malignant lymphoma of the prostate. This case report provides further insight into the diagnosis and therapy of MCL.

\section{References}

1. Sander B: Mantle cell lymphoma: recent insights into pathogenesis, clinical variability, and new diagnostic markers. Semin Diagn Pathol 28: 245-55, 2011.

2. Abdussalam A and Gerridzen RG: Mantle cell lymphoma of the prostate. Can Urol Assoc J 3: E94-E95, 2009.

3. Genkin I: Mantle cell lymphoma of prostate. Commun Oncol 3 : 69, 2006.

4. Chim CS, Loong F, Yau T, Ooi GC and Liang R: Common malignancies with uncommon sites of presentation: case 2. Mantle-cell lymphoma of the prostate. J Clin Oncol 21: 4456-4458, 2003.
5. Abrahamsson A, Dahle N and Jerkeman M: Marked improvement of overall survival in mantle cell lymphoma: a population based study from the Swedish Lymphoma Registry. Leuk Lymphoma 52: 1929-1935, 2011.

6. Smedby KE and Hjalgrim H: Epidemiology and etiology of mantle cell lymphoma and other non-Hodgkin lymphoma subtypes. Semin Cancer Biol 21: 293-298, 2011.

7. Yatabe Y, Suzuki R, Tobinai K et al: Significance of cyclin D1 overexpression for the diagnosis of mantle cell lymphoma: a clinicopathologic comparison of cyclin D1-positive MCL and cyclin D1-negative MCL-like B-cell lymphoma. Blood 95: 2253-2261, 2000

8. Ek S, Dictor M, Jerkeman M, Jirström K and Borrebaeck CA: Nuclear expression of the non B-cell lineage Sox11 transcription factor identifies mantle cell lymphoma. Blood 111: 800-805, 2008.

9. Xu W and Li JY: SOX11 expression in mantle cell lymphoma. Leuk Lymphoma 51: 1962-1967, 2010.

10. Le Gouill S, Mohty M, Guillaume T, Gastinne T and Moreau P: Allogeneic stem cell transplantation in mantle cell lymphoma: where are we now and which way should we go? Semin Hematol 48: 227-239, 2011.

11. Dietrich S, Tielesch B, Rieger M, et al: Patterns and outcome of relapse after autologous stem cell transplantation for mantle cell lymphoma. Cancer 117: 1901-1910, 2011. 